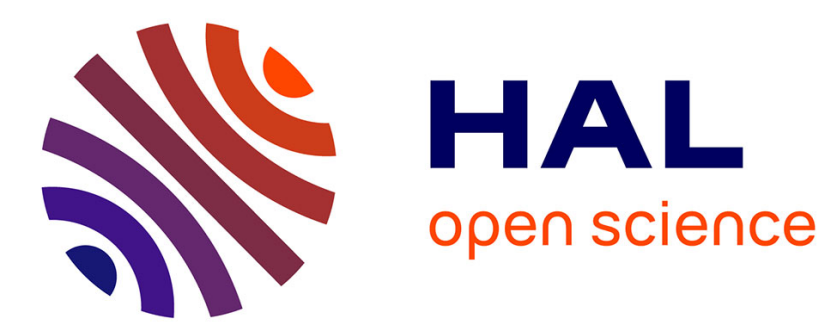

\title{
Towards a smart city based on cloud of things, a survey on the smart city vision and paradigms
}

Riccardo Petrolo, Valeria Loscrì, Nathalie Mitton

\section{To cite this version:}

Riccardo Petrolo, Valeria Loscrì, Nathalie Mitton. Towards a smart city based on cloud of things, a survey on the smart city vision and paradigms. Transactions on emerging telecommunications technologies, 2015, pp.1-11. 10.1002/ett.2931 . hal-01116370

\section{HAL Id: hal-01116370 \\ https://inria.hal.science/hal-01116370}

Submitted on 17 Nov 2015

HAL is a multi-disciplinary open access archive for the deposit and dissemination of scientific research documents, whether they are published or not. The documents may come from teaching and research institutions in France or abroad, or from public or private research centers.
L'archive ouverte pluridisciplinaire HAL, est destinée au dépôt et à la diffusion de documents scientifiques de niveau recherche, publiés ou non, émanant des établissements d'enseignement et de recherche français ou étrangers, des laboratoires publics ou privés. 


\title{
Towards a Smart City based on Cloud of Things, a survey on the smart city vision and paradigms
}

\author{
Riccardo Petrolo, Valeria Loscrì, Nathalie Mitton \\ Inria, Villeneuve d'Ascq, FRANCE \\ email: firstname.lastname@inria.fr
}

\begin{abstract}
Smart City represents one of the most promising, prominent and challenging Internet of Things (IoT) applications [22]. In the last few years, indeed, the smart city concept has played an important role in academic and industry fields, with the development and deployment of various middleware platforms and IoT-based infrastructures. However, this expansion has followed distinct approaches creating, therefore, a fragmented scenario, in which different IoT ecosystems are not able to communicate between them. To fill this gap, there is a need to re-visit the smart city IoT semantic and offer a global common approach. To this purpose, this paper browses the semantic annotation of the sensors in the cloud, and innovative services can be implemented and considered by bridging Cloud and Internet of Things. Things-like semantic will be considered to perform the aggregation of heterogeneous resources by defining the Cloud of Things (CoT) paradigm. We survey the smart city vision, providing information on the main requirements and highlighting the benefits of integrating different IoT ecosystems within the cloud under this new CoT vision.
\end{abstract}

This paper also discusses relevant challenges in this research area.

${ }^{*}$ Correspondence

Inria, Villeneuve d'Ascq, FRANCE

email: firstname.lastname@inria.fr

\section{INTRODUCTION}

Cities are growing steadily and urban living poses major challenges in our daily lives. As of $2007,50 \%$ of the world population was living in cities rather than rural areas. The United Nations Population Fund forecasts that by 2030 approximately $60 \%$ of the world population will live in an urban environment [30]. In this context, Information and Communication Technologies (ICT) together with local governments and private companies, play a key role for implementing innovative solutions, services and applications to make smart cities a reality. In this context the Internet of Things (IoT) paradigm is playing a primary role as an enabler of a broad range of applications, both for industries and the general population. The increasing popularity of the IoT concept is also due to the constantly growing number of very powerful devices like smartphones, tablets, laptops and lower powerful devices like sensors that are able to join the Internet. In the context of Smart Cities, it makes sense to consider the scenario of the various different and heterogeneous devices, the Wireless Sensor Networks interconnected to each other and to exploit these "interconnections" to activate new type of services. The ICT trends suggest that the sensing and actuation resources can be involved in the Cloud and solutions for the convergence and evolution of IoT and cloud computing infrastructures arise. Nevertheless, there are some challenges that need to be faced such as: 1) the interoperability among different ICT systems; 2) a huge amount of data to be processed provided in real-time by the IoT devices deployed in the smart systems; 3) the significant fragmentation deriving from the multiple IoT architectures and associated middleware; 4) heterogeneous resources mashup, namely how to orchestrate resources of the various Clouds. Concerning the last item, the concept of IoT, with underlying physical objects abstracted according to thing-like semantics, seems a valid starting point for the orchestration of the various resources. In this context, the Cloud concept could play the role to connect the IoT with the Internet of People through the Internet of Services, by the means of an horizontal integration of various silos. We will refer to this horizontal integration and to the Cloud computing associated to the IoT as the Cloud of Things. As we will see, this concept goes beyond to the interconnection and hyperlink of things. In this paper, we will explain how abstraction, virtualization and management of things have to be properly designed and implemented in order to develop solutions for the convergence of diverse IoT platforms and Clouds. A precise design of these mechanisms will permit 
the development of a technological-agnostic architecture, where the integration and deployment of diverse devices and objects can be considered by neglecting their underlying architecture. We will present the VITAL project as a Cloud of Things-based architecture, able to meet many critical requirements of a smart city, and we will show how this platform can be considered to bridge different and heterogeneous IoT silos. A preliminary description of the VITAL platform has been presented in [38] and [37], where the authors just introduce the platform and the Cloud of Things paradigm. In this work, we will give more details about the services that VITAl implements and we will describe specific use cases where the VITAL platform plays a very key role. The paper is structured as follows: in Section II we revise the literature regarding the IoT platforms. Section III introduces the concept of Cloud of Things (CoT), starting from the traditional Cloud computing concept. Section IV is devoted to present the main requirements of a city to be considered smart. In Section $\mathrm{V}$ we consider both paradigms, the IoT and the CoT, as potential effective solutions to make the cities smarter and more sustainable, based on the specific requirements individuated in Section IV. In Section VI we describe the VITAL platform as a CoT-based platform and effective solution to be applied for the realization of a smart city. In the Section VII, we give some practical examples of VITAL application, by defining some use cases. In the Section VIII, we describe the main ICT actors that the deployment of a Smart City implies. Finally, Section IX concludes the paper by investigation open research directions.

\section{IOT PLATFORMS}

"The Internet of Things has the potential to change the world, just as the Internet did. Maybe even more so" [3]. With this sentence, in 1998, Kevin Ashton introduced for the first time the term "Internet of Things" (IoT). Some years later, in 2005, the International Telecommunication Union (ITU) formally introduced IoT, according to which: "from anytime, anyplace connectivity for anyone, we will now have connectivity for anything" [26].

Since then, IoT starts to be a hot topic in academic and industry fields. Several EU projects have been launched with the goal to provide solutions for the realization of the IoT and its integration in different application domains. The main reasons behind this interest are the capabilities that the IoT will offer. It promises to create a world where all the objects around us are connected to the Internet and communicate with each other with minimum human intervention [35]. These potentialities make possible the development of a huge number of applications in different domains as shown in Figure 1 according to [4].

At the same time and together with the several IoT platforms, the middleware started to gain more and more importance. Middleware gained in the last years a lot

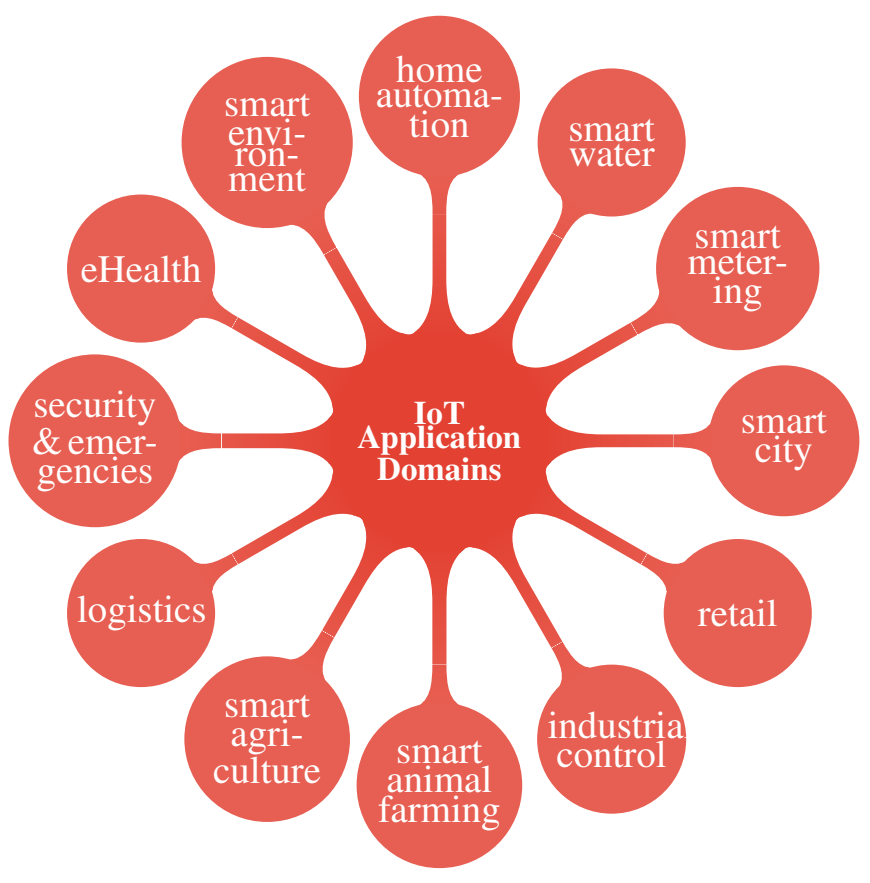

Figure 1. IoT Application Domains [4].

of importance due to their major role in simplifying the development of new services and the integration of legacy technologies into new ones [5]. In the following, we will present some of the most representative IoT platforms without pretending to be exhaustive:

$G S N$ [19] is a platform developed in Java, aiming at providing flexible middleware to address the challenges of sensor data integration and distributed query processing. It lists all the available sensors in a combo-box which users need to select the ones they need. GSN's purpose is to make the GSN applications hardware-independent and the changes and variations invisible to the application. The main limitation of GSN is that it lacks semantics to model the metadata.

LSM [28] (Linked Sensor Middleware) is a platform that brings together the live real world sensed data and the Semantic Web. It provides many functionalities such as, wrappers for real time data collection and publishing; a web interface for data annotation and visualization; and a SPARQL endpoint for querying unified Linked Stream Data and Liked Data.

Sensor-Cloud [53] is an infrastructure that aims at managing physical sensors by connecting them to the cloud. This infrastructure provides the service instances (virtual sensors) to the users in an automatic way at the same fashion as these virtual sensors are effectively part of the IT resources. The generation of the services instances implies that the sensor devices and service templates (used to create the virtual sensors) should be firstly prepared. 
It uses SensorML to describe the metadata of physical sensors. It just focuses on managing sensors via cloud.

OpenIoT [31] represents a joint effort of several contributors to IoT-based applications according to a cloud computing delivery model. It concentrates on providing a cloud-based middleware infrastructure in order to deliver on-demand access to IoT services, which could be formulated over multiple platforms. Its middleware infrastructure permits to collect and filter data information from the Internet-connected objects, by the means of opportunistic algorithms. It is strictly related to the FP7 VITAL project [46].

Xively [52] (formerly known as Cosm and Pachube) offers a public cloud for the IoT that simplifies and accelerates the creation, deployment and management of sensor in scalable manner.

As we will better explain, these approaches strengthen the vision towards the Cloud of Things, but a lot of new enhancements are still needed to realize a Cloud of Things (CoT) platform for Smart City.

\section{TOWARDS CLOUD OF THINGS}

Cloud Computing attracts the attention from both academy and industry across the world, because it is capable of transforming service provision models over the entirely current IT (Information Technology) industry. It provides criterion for service provisions with reduced upfront investment, expected performance, high availability, tremendous fault-tolerance capability, infinity scalability, and so on [54].

According to [33] the services can be divided in three layers (Figure 2):

- Infrastructure as a Service (IaaS) which offers computing resources such as processing or storage;

- Platform as a Service (PaaS) designed for software developers, in order to allow them to write their applications according to the specifications of a particular platform without needing to worry about the underlying hardware infrastructure;

- Software as a Service (SaaS) is the most visible layer of Cloud Computing for end-users, because it is about the actual software applications that are accessed and used.

In addition to the above main layers, some others are also introduced and discussed in literature such as Data as a Service (DaaS), Network as a Service (NaaS), Identity and Policy Management as a Service (IPMaaS), and so on. In [6] authors introduce XaaS (everything as a service model) that promotes the "pay as you go" method, allowing the consumers to consume a service from a service provider by paying only for the amount of resources they actually use. Within the Internet of Things context, this approach gave the input to the so-called Cloud of Things (CoT) [16], which deals to implement indexation and querying services of things, and provide them to

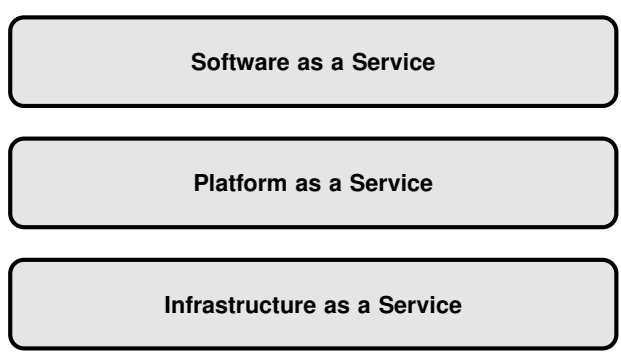

Figure 2. Cloud Computing service models [33].

final users, developers, provides, as a service (Figure 5). One interesting model to enable CoT is comprehensively discussed by authors in [36]. They focus indeed, on the Sensing as a Service model based on Internet of Things (IoT) infrastructure, which introduces four conceptual layers:

- Sensor and Sensor Owners Layer that consists of sensors and how the owner manages them, allowing or not, the publication in the cloud.

- Sensor Publishers that detect available sensors, communicate with the sensor owners, and get permission to publish sensors in the cloud.

- Extended Service Providers which communicates with multiple Sensor Publishers in order to select sensors based on costumer's requirements.

- Sensor Data Consumers that need to register themselves in order to consume sensors data.

The advantages and benefits promised by the Sensing as a Service model are numerous, and just to name the majors we have: sharing and reusing of sensor data (if someone has already deployed the sensors, others can have access to them by paying a fee to the sensor owner), reduction of data acquisition cost due to the shared nature, collect data previously unavailable (thanks to the business model, companies are stimulated to "sell" them sensors data).

In the last few years, researchers have mainly focused on representing the observation and measurement data from sensor networks, according to the Sensor Web Enablement (SWE) proposed in [11] by the Open Geospatial Consortium (OGC). However, these standards do not provide facilities for abstraction, categorization, and reasoning rather offered by [14], within the W3C Semantic Sensor Network Incubator group (SSN-XG) defined an OWL2 [23] ontology, answering the need for a domain-independent and end-to-end model for sensing applications by merging sensor-focused (e.g. SensoML [11]), observation-focused and system-focused views. It has received consensus from the community and has been adopted in several projects like Spitfire EU Project *.

*http://spitfire-project.eu 
Regarding the heterogeneity in communications technologies, interesting solutions for resource-constrained devices (e.g. sensors) are provided by 6LoWPAN [18] and CoAP [15].

6LoWPAN enables the integration of sensors to the Internet thanks to mechanisms which allow transmission of IPv6 packets. In order to convert IPv6 packets to 6LoWPAN and vice versa, a gateway (i.e. border router) is required. It handles the necessary tasks such as header compression and enables the seamless usage of IPv6 across the heterogeneous network architectures. 6LoWPAN has been developed in an early stage for the TinyOS, Contiki, and FreeRTOS operating systems for WSNs [29].

CoAP is an application layer protocol designed for energy constrained devices. It deals with Constrained RESTful Environments (CoRE) [48], providing a lightweight alternative to HTTP. Devices supporting CoAP provide flexible services over any IP network using UDP. Any HTTP client or server can interoperate with CoAPready endpoints by simply installing a translation proxy between the two devices [13].

Summing up, IoT devices can be connected to the Internet, their data can be annotated using a sensor ontology (i.e., SSN ontology), encoded in standard Web formats (i.e., RDF), and made available on the Cloud, establishing therefore the Cloud of Things.

\section{SMART CITY REQUIREMENTS}

Before to relate the IoT and CoT paradigms to what is considered as a very emerging and important application domain, namely Smart City, it is useful to identify the main requirements, in terms of ICT-based services and solutions, that a city presents. To this purpose, by following the same approach introduced in [2], we make reference to two different types of requirements, that are: 1) service/application, considered from the point of view of the citizens and 2) operational, seen from the city authorities and administrators of the networks viewpoint. Concerning the service/application aspects, the end-users devices equipped with multiple radio technologies and several sensors and actuators deployed all over the cities, make possible the individuation of novel services and applications for the citizens. These services will have specific features, like: a) user-centric: based on the specific context and the preferences of the users, b) ubiquitous: reachable everywhere and from any devices, and c) highly-integrated: based on the integration of services and data from several and different applications or on the social cooperation of multiple users. Of course, beyond the citizens, also the stakeholders of a city, like educational institutions, healthcare and public safety providers, governmental organizations, etc. will be in conditions to exploit the key features of these new services that make the city more sustainable. On the other hand, the Smart City concept considered from the point of view of the administrations and the networks providers are translated into a network infrastructure that is: a) highlyinterconnected: by overcoming the heterogeneity of the devices and the IoT platforms, it is possible to provide ubiquitous connectivity, b) cost-efficient: the deployment and organization of the network should be as much automatic as possible and independent from the human intervention, c) energy-efficient, able to realize an efficient resource utilization, in order to meet the main requirements of green applications d) reliable: that connectivity, the ubiquity of the network should be guaranteed above all in the case of exceptional and adverse conditions. The real scenario we can observe at the moment, is characterized with a high level of fragmentation of technologies, lack of ubiquity in terms of both connectivity and coverage, due to the plethora of technologies and devices present in a city. This fragmentation is mainly due to the presence of many access networks usually managed by different operators (i.e. Universal Mobile Telecommunications System UMTS, Worldwide Interoperability for Microwave Access - WiMAX, WiFi, etc.). Even if some steps ahead have been moved thanks to some projects, most of these initiatives are related to specific cities and do not consider general architectures. By considering the main IoT platforms and the CoT concept, we will try to explain how the main requirements of a city to become a smart city can be fulfilled and at the end we will show how the VITAL platform can play the role of "interconnecting" heterogeneous ICT silos and devices.

\section{IOT AND COT FOR A SMART CITY}

Among all the domains that can take advantages from the IoT platforms, the Smart City concept received in the last few years a significant research effort and technological development. The most notable reason for these attentions, is attributable to the population growth and the urbanization trend. According to the United Nations, the urban populations will grow to an estimated 2.3 billion over the next 40 years, while as much as $70 \%$ of the world's population will live in cities by 2050 . The rapid growth of cities became the main driver of global environmental changes, as cities, occupying only $2 \%$ of the earth landmass consume about $75 \%$ of the world's energy and produce $80 \%$ of its greenhouse gas emission. Such a dramatic expansion of the cities has brought to focus the need to develop cities in a sustainable manner, while also making the quality of life in the cities better [50].

Although there is not a formal and universally accepted definition of "Smart City" yet, in [20], authors try to delineate the concept, defining a Smart City as a city which functions in a sustainable and intelligent way, by integrating all its infrastructure and services into a cohesive whole and using intelligent devices for monitoring and control, to ensure sustainability and efficiency. 
This interpretation makes evident, therefore, that Smart City concept (Figure 3) needs interoperability between the different IoT deployments that are, today, mainly closed and vertically integrated to specific application domains [45].

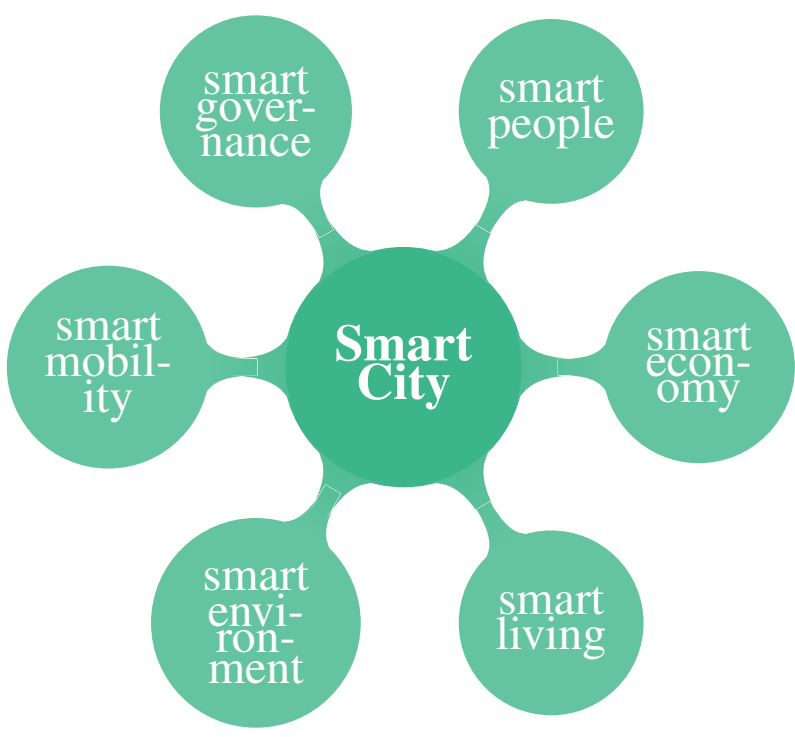

Figure 3. Smart City concept.

These solutions are, indeed, based on multiple architectures, standards and platforms, which have led to a highly fragmented IoT landscape and make challenging the realization of the Smart City concept. According to [27], we can recognize the IoT structure divided into five layers (Figure 4):

- Device Layer that deals with the identification and collection of objects specific information by the sensor device;

- Network Layer used to send data collected by the Device Layer to the information processing system;

- Middleware Layer that performs information processing and ubiquitous computation and takes automatic decision based on the results;

- Application Layer which provides global management of the application based on the information processed through the Middleware;

- Business Layer that is responsible for the management of overall IoT system.

Within the context of Smart City [12], the Cloud of Things is expected to play a significant role. CoT indeed, can make better use of distributed resources, puts them together in order to achieve higher throughput and be able to tackle large scale computation problems [43], enabling therefore, the horizontal integration of various (vertical) Internet of Things platforms and so the Smart City vision. Moreover, it allows users to express the service they want providing the relevant data back to them quickly without

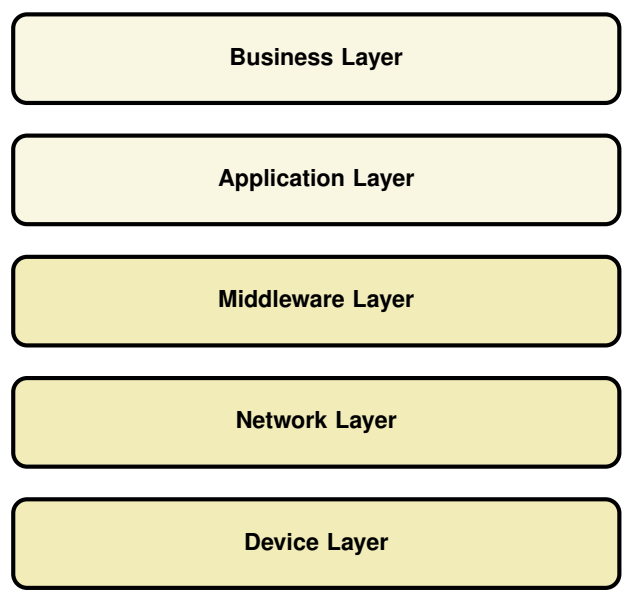

Figure 4. The loT Architecture [27].

asking the users to manually select the sensors which are relevant to their requirement.

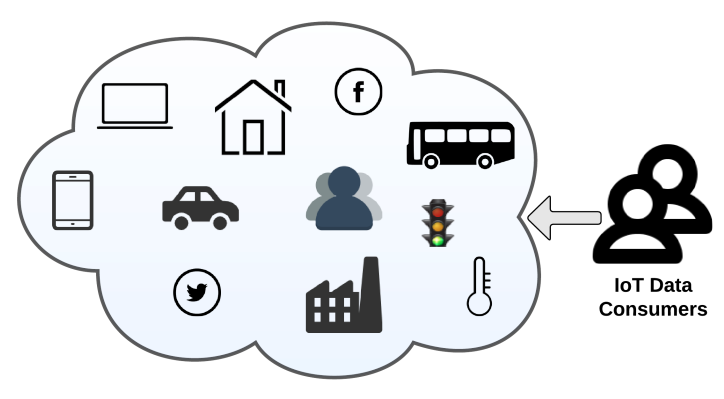

Figure 5. Cloud of Things.

Certainly, CoT needs to deal with several challenges, but the major in terms of research regards the heterogeneity in sensor types (e.g., temperature, NFC, RFID), and in protocols and communications technologies (e.g. Wi$\mathrm{Fi}$, ZigBee); interoperability among different sensors hardware and cloud solutions. It is therefore important to define an abstraction level, in order to bridge the gap between the disparate technologies. Regarding the various sensor type, the use of the technologies developed in the semantic web [9] such as ontologies, semantic annotation, linked data [8] and semantic web services has recently gained momentum in this field. These technologies promote interoperability among IoT resources, information models, data providers and consumers and simplifies effective data access and integration, resource discovery, semantic reasoning, and knowledge extraction [7]. 


\section{VITAL as A CoT-based SMART CITY PLATFORM}

One of the most important objectives of VITAL is about the integration of Inter-Connected Objects (ICOs) among multiple IoT platforms and ecosystems. The project explores the convergence and federation of multiple IoT platforms by taking account of cost efficiency of the deployments. In the context of VITAL, a very key factor is represented by the virtualization of interfaces that in combination with cross-context tools that enable the access and management of heterogeneous objects supported by different platforms and managed by different administrative stakeholders let us to define the VITAL platform as a Cloud of Things architecture. In Figure 6 we show an overview of the VITAL platform.

As we can observe in Figure 6, the data and services access of the heterogeneous objects involved in VITAL, is based on the implementation of the VUAIs (Virtualized Universal Access Interfaces), that makes possible to consider a single virtual access by making the architecture platform-agnostic. These key features of VITAL make this platform able to embrace the CoT philosophy. The VUAI layer is built upon a so-called meta-architecture and migration layer and includes several connectors to communicate and interconnect different IoT platforms and clouds. In practice, this module deals with issues related to the management of the overall VITAL infrastructure built on top of existing IoT architectures and cloud platforms and enables heterogeneous mashup. The VUAIs allow the implementation of a kind of abstraction, where "objects" handler that point to physical items, can be discovered, selected and filtered and also allocated by following a "Things as a Service" (TaaS) paradigm. In this sense, the VITAL as CoT platform is something that goes beyond the interconnection and hyperlinking "things" of the IoT paradigm. VITAL also includes a datastore for data like geographical information and smart city stakeholders. Of course, it is expected that the management of this kind of information giving location awareness and other context related information can be effectively exploited in the optimization of computing and sensing of the management of the various clouds. The CoT paradigm implies the implementation of querying services and indexing of things, the aggregation of heterogeneous resources based on a given thing-like semantics and provided to the final stakeholder (final user, developer, etc.). Moreover, the CoT concept explicitly has to consider mechanisms to abstract, virtualize and manage things as performed in VITAL. It is worth outlining that VITAL is based on W3C SSN ontology, that is considered ideal as a basis for unifying the semantics of different IoT platforms, since it is domain independent and extensible. Several additional concepts have to be considered to enhance the ontology starting from information about city-wide, stake-holders, IoT system, etc. The ontology update with additional functionalities will allow the migration of smart city application across different urban environments.

\section{VITAL ontologies}

The main challenge of VITAL is to integrate in a platform agnostic way a multitude of heterogeneous data and functionalities produced and offered by different sensors and IoT systems managed along by disparate (independent) organisations and entities. This can be achieved thanks to Linked Data functionalities, which aim to describe and integrate data provided by different organisations in an interoperable way [21]. VITAL indeed relies on Linked Data standards (i.e., RDF, JSON-LD, and ontologies) for modeling and accessing data; in particular, ontologies are used to specify data formally. As shown in Table I, we reuse as many ontologies as possible, while new ontologies need to be exploited to model relevant Smart Cities entities and for describing the VITAL system itself.

The VITAL ontologies can be grouped into four main areas - sensors and sensor measurements, Smart Cities, IoT systems and services, VITAL systems and services that we describe in the following.

Sensor and sensor measurements represent a crucial part of the VITAL data model. Many ontologies have been already developed in the field, and are based on multiple layers of abstraction [49]. It is important to clarify that since there are many application-specific cases, it is impossible to specify a single ontology that defines the semantics of all possible data items. VITAL combines several ontologies. It uses Semantic Sensor Network (SSN) [14] as a generic sensor ontology since it permits to describe sensors, including their accuracy and capabilities, observations, methods for sensing, concepts for operating and survival ranges, and deployments. Beyond this, in order to describe other basic concepts like time, location and unit of measurement, VITAL uses wellknown ontologies [24, 25, 40].

Smart Cities have many capabilities that must be modeled as part of a smart city ontology. According to the VITAL use case as defined in Section 7, the most relevant producers of data come under the following headings:

- transport - e.g. dynamic route calculation informed of accidents and congestion;

- energy - e.g. reporting of faults;

- emergency services - e.g. detection of accident and crimes;

- waste management - e.g. detection of full bins;

- air and water - e.g. reporting of air and water pollution;

- recreation - e.g. produce data on large events such as concerts to inform public transport. 


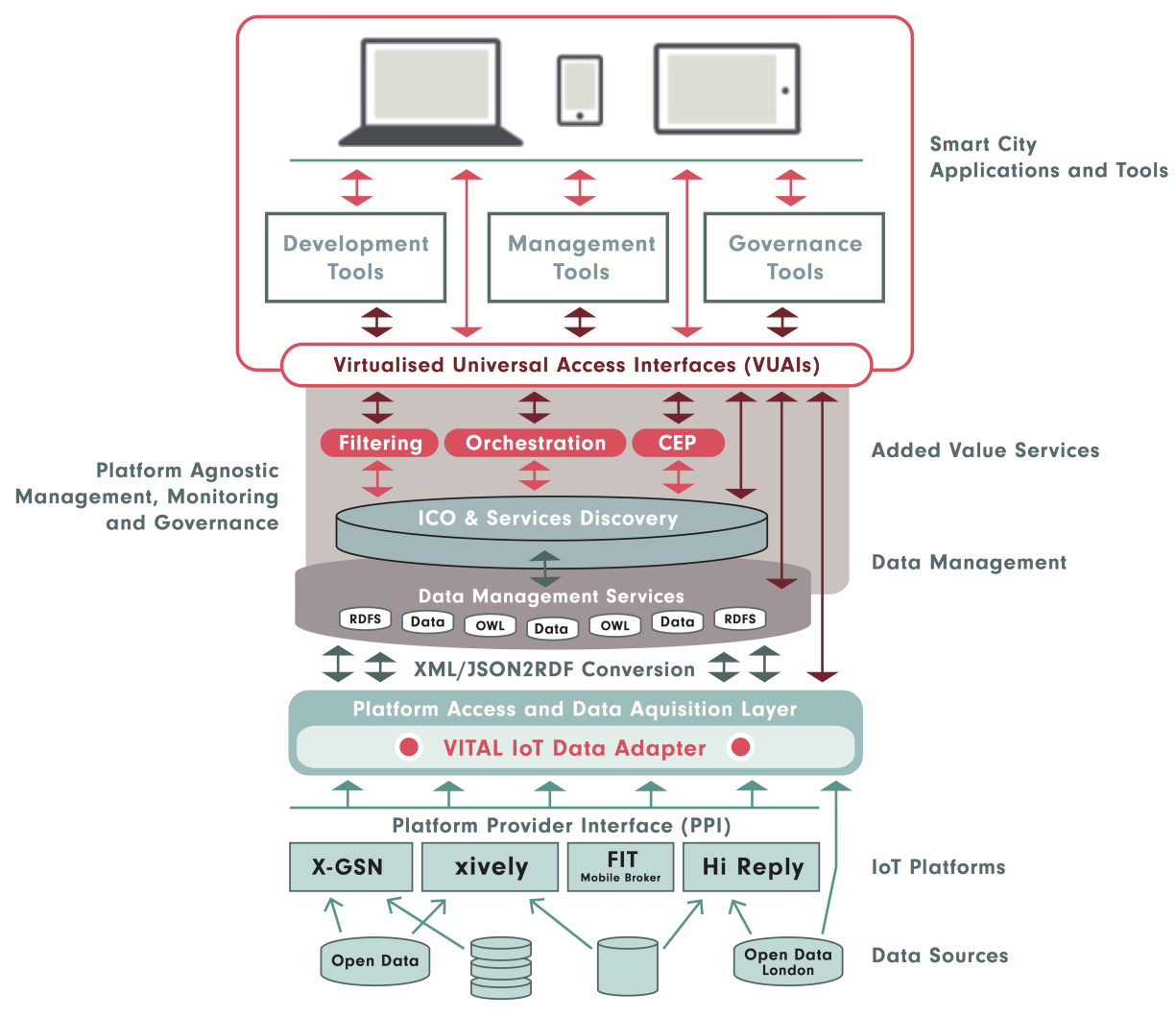

Figure 6. VITAL Platform

The majority of the VITAL semantic information on cities are obtained via the classic DBpedia ${ }^{\dagger}$ dataset. However, modeling Smart Transport is a non-trivial task since it covers a wide range of domains (e.g. tracking pedestrian congestion, smart traffic light systems, etc.). Therefore, VITAL uses, again, a combination of ontologies, and the core of them is the Ontology for Transportation Networks (OTN) [41] that allows an easy modeling of a transport network graph with connections between infrastructures (e.g., bus) as well as dynamic events such as accidents and blocked passages. It is important to emphasize that VITAL is not restricted to smart transport scenarios; a user who would like to adopt VITAL for other smart city aspects can do so by specifying additional ontology elements. Thanks to the nature of Linked Data indeed, additional elements can be added at any time without the need to redesign the whole system.

The integration of heterogeneous IoT systems and services represents another challenging point for the VITAL consortium. In order to model them, and in particular the four platforms so far chosen - GSN [19], Reply H1, FIT IoT-Lab [17], and Xively [52] - VITAL uses the SSN ontology. In this way, an IoT system is modeled as a subclass of ssn : System with a number of additional properties (e.g. operator, status, etc.). In addition, an IoT system may specify a set of IoT services offered.

Above the IoT systems aforementioned, we need also to model the VITAL system itself and its services. From the semantic point of view, a VITAL system is similar to an IoT system, therefore we decided to model it as a subclass of IoT System.

\section{VITAL'S USE CASES}

In this section, we describe a potential VITAL use case, where the concept of the different services that characterize VITAL (i.e. Discovery, Filtering , Orchestration and Complex Event Processing (CEP)) play a key role. Note that more use cases are available on the project website $\ddagger$ and in the project deliverables.

\footnotetext{
†http://dbpedia.org/
} 
Table I. VITAL ontologies

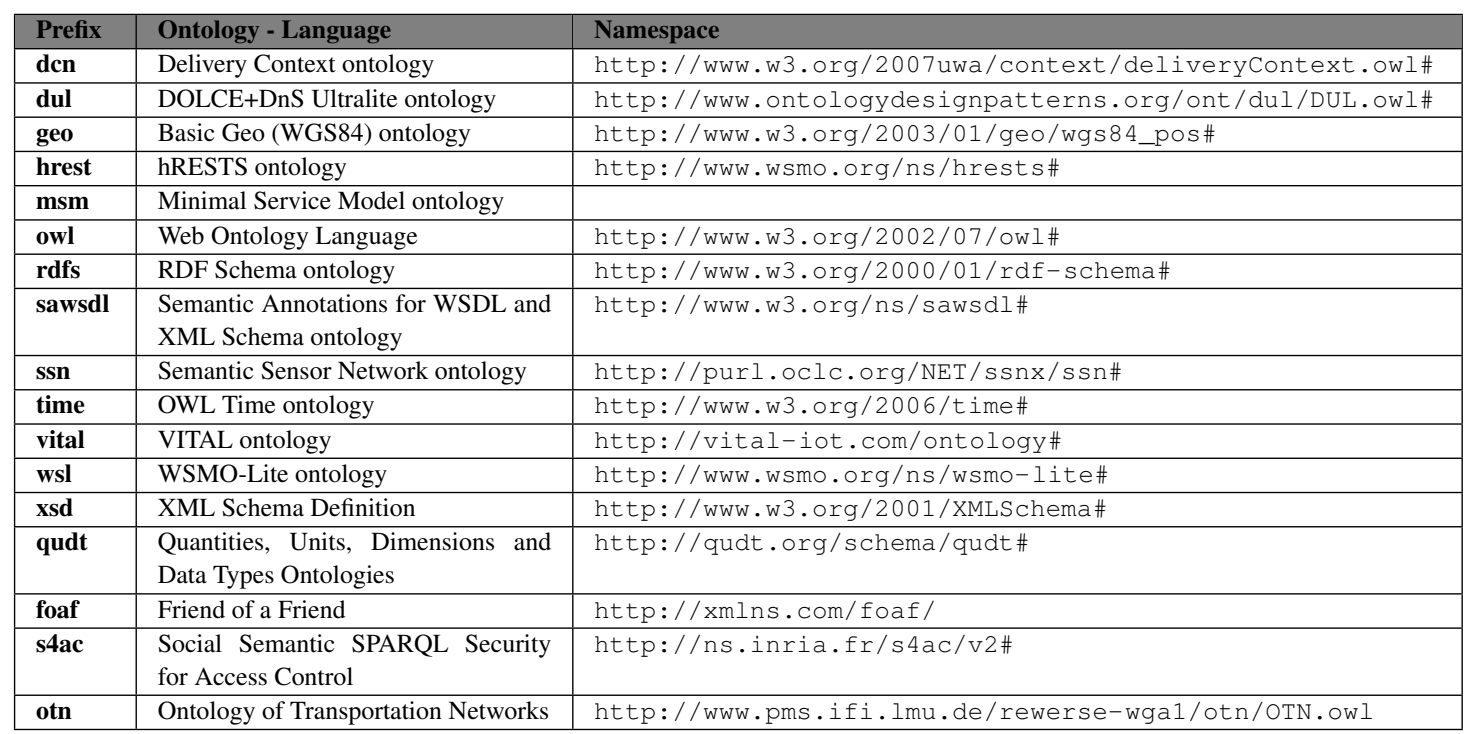

\subsection{Use Case 1: VITAL for Smart Street Management}

An effective management of the roads would make easier the citizens' life and would also have an economic impact. Just as an example, we could consider Public Lightening Management, which represents a significant source of energy wasting. In this context, the VITAL platform "reinterprets" every light source (each equipped with sensors) as an Internet-Connected Object (ICO). The VITAL services (e.g. Discovery, Filtering, Orchestration and CEP) allow an efficient management of the information, through the interconnection of data originated from different clouds. This intelligent manipulation of the data will be exploited in several ways such as: 1) it will be translated in "actuation" for an automatic regulation of the luminous intensity; 2) it will be used to infer useful information such as the actual schedule of public transportation (e.g. if a bus is incoming and the exact number of the bus); 3) it will be used for the intelligent management of the trash containers (e.g. if a trash container has to be emptied), etc. Concerning the intelligent management of the trash container, the VITAL platform could be very effective. In fact, the discovery of the areas where the trash containers are deployed and the filtering of the zones where an immediate intervention is necessary (e.g. a trash container that needs to be emptied), represent just an example of intelligent and dynamic manipulation and actuation deriving from the VITAL architecture. From this very simple use case scenario, it is also possible to understand that data in the cloud could help the stakeholders, by redefining the deployment of the trash containers dynamically. In practice, the concept of services as defined in the VITAL platform is to allow the manipulation and management of data coming also from zones that are geographically far to each other also through the definition and implementation of complex algorithms.

\subsection{Use Case 2: VITAL for Smart Traffic Management}

In the last years, the technological advancements allowed a very deep diffusion of complex GPS navigators. Several drivers make use and exploit these tools, that give very valuable information on the traffic conditions also in real time. They are also very valuable in the case of accidents alerts. Anyway, also in this context, the VITAL platform, through the definition and implementation of its Cloud of Thing paradigm, is able to play a key role. Let us refer to a scenario where an accident is broadcast/"alerted" (e.g. through the use of a GPS navigator). Normally, after such an alert occurs, the "minor" roads are taken as viable alternative solutions, with the side effect that they will be soon overcrowded. Moreover, this type of navigators do not take special events into consideration, such as concerts or festivals. The elaboration of data coming from different ICOs allows a smarter elaboration of different kinds of information. In this sense, the VITAL platform does not limit its "intervention" to a simple alert and alternative roads, but the VITAL services will output alternatives that consider many different "aspects" and the final user will play an active and aware role by choosing based on different type of info.

\section{TECHNOLOGIES}

Deploying a Smart City is a multi-disciplinary task, that involves various stakeholders from different thematic 
areas, like politics, finance, city management and organization, and information and communication technologies (ICT). From the ICT perspective, the number of devices, technologies, and standards involved is huge, therefore, it is necessary to have an overall view. In the next subsections we summarize the main technology's actors.

\subsection{Devices}

Sensors represent an essential component of any intelligent control system. Thanks to technology advances today a multitude of different sensors is available, enabling applications that were unimaginable in the past. From the perspective of the requirements for Smart Cities, wide availability of these technologies translates to a large number of opportunities in terms of sensing, i.e., meters to determine gas, electricity, water etc.

Sensor nodes are usually scattered in a sensor field; each of these scattered sensor nodes has the capabilities to collect data and route data back to a special node called sink by a multi-hop infrastructureless architecture [1]. Currently, most of commercial Wireless Sensor Network (WSN) are based on the IEEE 802.15.4 standard, which defines the physical and MAC layers for low-power, low bit-rate communications in wireless personal area networks (WPAN). However, IEEE 802.15.4 does not include specifications on the higher layers of the protocol stack, which is necessary for the seamless integration of sensor nodes into the Internet [5]. The role of SN in the IoT is crucial and well examined in [35], wherein authors point out on the relation between IoT and Sensor Networks: $S N$ can exist without the IoT, however, the IoT cannot exist without $S N$.

Smartphones represent another interesting sensing scenario. Indeed, the ever increasing number and the presence of a mixture of sensors such as GPS, gyroscopes, accelerometers and compasses, enabling a variety of crowd sourcing applications, which will eventually be augmented by the IoT. For instance, as users regularly update their location status on social networks like Twitter and Facebook, based on this location information, it is possible to aggregate this data, enabling tasks to be dispatched to people in specific locations [20].

Regarding to short range communications, two interesting technologies in the smart cities context are Radio Frequency Identification (RFID) and Near Field Communication (NFC).

RFID is a method of identifying unique items using radio waves. Typical RFID systems are made up of 2 major components: readers and tags. The reader sends and receives RF data to and from the tag via antennas. The tag is made up of the microchip that stores the data, an antenna, and a carrier to which the chip and antenna are mounted [47]. RFID can be used to develop a large number of Smart City applications, to name a few: smart parking [32], traffic monitoring [34], library management [42], transportation tickets, etc.
NFC is a contactless wireless communication technology based on RFID and Internet technologies [51]. It supports the communication within $20 \mathrm{~cm}$, and it represents a prominent technology in the Smart City scenario, enabling a range of applications in order to make people's life more convenient and fast, i.e. the digital wallet introduced in [44] or building access control.

\subsection{Networking}

Integrating resource-constrained devices into the Internet is difficult since ubiquitously deployed Internet protocols such as HTTP, TCP, or even IP are too complex and resource-demanding [39].

6LoWPAN [18] defines mechanism which allows the transmission of IPv6 packets. CoAP [15] is an application layer protocol designed for energy constrained devices. It deals with Constrained RESTful Environments, providing a lightweight alternative to HTTP. CoAP and 6LoWPAN allow sensor nodes to be integrated into the web, through the use of proxies for HTTP to CoAP conversion.

However the short range transmission problem still exists. Regarding the Link Layer, Smart City system requires a set of link technologies that can easily cover wide geographical area and support, at the same time, a possibly large mount of traffic.

\section{CONCLUSION AND FUTURE CHALLENGES}

In this paper, we have considered the IoT platforms as a viable solution to make cities smarter. We have shown how the proliferation of ICT represents new opportunities for the development of novel services, contributing to make the cities more sustainable. The different IoT platforms, the various IoT clouds and the several IoT applications and services have resulted in different and heterogeneous IoT ecosystems, that introduce a significant degree of fragmentation. We presented several IoT platforms that can be efficiently considered in the context of Smart City, but in order to bridge the gap between the different IoT platforms it is necessary to consider a convergence of these platforms and ecosystems. In this paper we envisaged in the Cloud computing a valid bridge of the IoT, Internet of people through the Internet of Services. This novel perspective allows the realization of an horizontal integration of various vertical platforms. Through the implementation of a specific virtualization level, the VITAL platform in the context of the correspondent FP7 EU project, ensures the semantic interoperability of various and different IoT platforms. The Virtualized Unified Access Interfaces (VUAIs) implement a meta-architecture and migration layer, with different connectors and drivers to permit the communication among the various platform. In this way, VITAL CoT-based platform can be considered as a very promising solution for the fragmentation issues in the context of Smart Cities. 
There are different challenges related to the Cloud of Things in smart cities, from both technical and privacy points of view.

Big Data. The overall IoT data produced by things is growing up fast, becoming really big data. Within this context, the challenges can be divided in two main categories [10], engineering in order to perform data management activities such as query, and storage efficiently; and semantic, in order to extract the meaning of the information from massive volumes of data.

Privacy \& Security. Privacy and Security issues exist for a long time in the computing literature, and many law acts have been published to protect users [54]. Certainly the main problematic within the CoT context is to define mechanisms in order to let the decision to publish or not data to "sensor owners". Other issues may come from the cyber-crime, indeed the system can be prone to cyberterrorism and cyber-vandalism.

\section{ACKNOWLEDGMENT}

This work is partially supported by CPER Nord-Pasde-Calais/FEDER CIA, the European Community in the framework of the VITAL FP7 project (Virtualized programmable InTerfAces for smart, secure and costeffective IoT depLoyments in smart cities under contract number FP7-SMARTCITIES-608662 and the Inria Project Lab SmartCities@Inria. The authors acknowledge help and contributions from all partners of the project.

\section{REFERENCES}

1. AKYILDiZ, I.F. AND SANKARASUBRAMANIAM, Y. AND CAYIRCI, E. A survey on sensor networks. IEEE Communications Magazine 40 (2002).

2. Aloi, GiAnluCA AND ET AL. STEM-Net: an evolutionary network architecture for smart and sustainable cities. Trans. on Emerging Telecommunications Technologies 25, 1 (2014).

3. Ashton, Kevin. That 'Internet of Things' Thing. RFID Journal (2009).

4. Asin, Alicia AND GAscon, David. 50 Sensor Applications for a Smarter World. http: //www. libelium.com/top_50_ iot_sensor_applications_ranking.

5. Atzori, Luigi AND IERA, ANTONio AND MoraBITO, Giacomo. The Internet of Things: A survey. Computer Networks 54, 15 (2010).

6. BANERJEe, Prith AND ET AL. Everything as a Service: Powering the New Information Economy. Computer 44, 3 (2011).

7. Barnaghi, Payam and Wang, Wei and HenSON, CORY AND TAYLOR, KERRY. Semantics for the Internet of Things. International Journal on Semantic Web and Information Systems 8, 1 (2012).
8. Berners-Lee, Tim. Linked data. W3C Design Issues (2006).

9. Berners-Lee, Tim And Hendler, JAmes And LASSILA, ORA. The semantic web. Scientific American 284, 5 (2001).

10. BIZER, ChristiAn AND ET AL. The meaningful use of big data. ACM SIGMOD Record 40, 4 (2012).

11. Botts, Mike and Percivall, George And REED, CARL AND DAVIDSON, JOHN. OGC Sensor Web Enablement: Overview And High Level Architecture. OGC White Paper 07-165 (2007).

12. Caragliu, A. And Del Bo, C. And Nijkamp, P. Smart cities in Europe. Tech. Rep. 0048, University Amsterdam, 2009.

13. Castellani, Angelo P. And Gheda, Mattia AND BUI, Nicola AND Rossi, Michele AND ZorZI, Michele. Web Services for the Internet of Things through CoAP and EXI. In Proceedings of Interntional Conference on Communications Workshops (2011).

14. Compton, Michael AND ET AL. The SSN ontology of the W3C semantic sensor network incubator group. Web Semantics: Science, Services and Agents on the World Wide Web 17 (2012).

15. Costantino, LuCA AND ET AL. Performance analysis of an LTE gateway for the IoT. In Proceedings of Interntional Symposium on a World of Wireless, Mobile and Multimedia Networks (2012).

16. Distefano, Salvatore and Merlino, GioVANNi AND PUliafito, ANTONio. Enabling the Cloud of Things. In Procedings of Conference on Innovative Mobile and Internet Services in Ubiquitous Computing (2012).

17. FIT IoT-Lab. https://www. iot-lab. info.

18. G. Montenegro and N. Kushalnagar and J. Hui AND D. CUller. Transmission of IPv6 Packets over IEEE 802.15.4 Networks, 2007.

19. GSN. https://github.com/LSIR/gsn.

20. Hancke, Gerhard P. AND Silva, Bruno De CARVALHO E. The role of advanced sensing in smart cities. Sensors 13, 1 (2013).

21. Heitmann, Benjamin and Kinsella, Sheila AND HAYES, CONOR AND DECKER, STEFAN. Implementing Semantic Web applications: reference architecture and challenges. In Proceedings of the 5th Workshop on Semantic Web Enabled Software Engineering (2009).

22. HeO, TAEWOOK AND ET AL. Escaping from ancient rome! applications and challenges for designing smart cities. Trans. Emerging Telecommunications Technologies 25, 1 (2014).

23. Hitzler, Pascal AND et AL. OWL 2 Web Ontology Language Primer. Tech. rep., World Wide Web Consortium, 2009.

24. Hobbs, J. R., And PAn, F. Time Ontology in OWL. Tech. rep.

25. Hodgson, Ralph and Keller, Paul J. AND 
Hodges, JACK AND SPIVAK, JACK. QUDT - Quantities, Units, Dimensions and Data Types Ontologies. Tech. rep.

26. ITU INTERNET REPORTS. The Internet of Things, 2005.

27. KHAN, R. AND ET AL. Future Internet: The Internet of Things Architecture, Possible Applications and Key Challenges. In Proceedings of Interntional Conference on Frontiers of Information Technology (2012).

28. LSM. http://lsm.deri.ie.

29. MAZZER, YANNIS AND TOURANCHEAU, BERNARD. Comparisons of 6LoWPAN Implementations on Wireless Sensor Networks. In Proceedings of Interntional Conference on Sensor Technologies and Applications (2009).

30. NAPHADE, M. AND BANAVAR, G. AND HARRISON, C. AND PARASZCZAK, J. AND MORRIS, R. Smarter cities and their innovation challenges. Computer 44 (2011).

31. OpENIoT. http://openiot.eu.

32. PAla, ZEydin AND InANC, NiHAT. Smart Parking Applications Using RFID Technology. In Proceedings of Annual RFID Eurasia (2007).

33. Patidar, Shyam and Rane, Dheeraj and Jain, Pritesh. A Survey Paper on Cloud Computing. In Proceedings of Second Interntional Conference on Advanced Computing \& Communication Technologies (2012).

34. Paul, Joydeep and Malhotra, Baljeet and Dale, Simon And QIang, Meng. RFID based vehicular networks for smart cities. In Proceedings of Interntional Conference on Data Engineering Workshops (2013).

35. Perera, C. And et Al. Context Aware Computing for The Internet of Things: A Survey. IEEE Communications Surveys \& Tutorials 16, 1 (2014).

36. Perera, Charith and Zaslavsky, Arkady B. and Christen, Peter and Georgakopoulos, DIMITRIOS. Sensing as a service model for smart cities supported by internet of things. Trans. Emerging Telecommunications Technologies 25, 1 (2014).

37. Petrolo, RicCARdo AND Loscri, VALERIA AND Mitton, Nathalie. Towards a cloud of things smart city. IEEE COMSOC MMTC E-Letter 9, 5 (2014).

38. Petrolo, Riccardo AND Loscri, VAleria And Mitton, NAthalie. Towards a Smart City based on Cloud of Things. In Proceedings of the International ACM MobiHoc Workshop on Wireless and Mobile Technologies for Smart Cities (2014).

39. PFISTERER, DENNIS AND ET AL. SPITFIRE: toward a semantic web of things. IEEE Communications Magazine 49 (2011).

40. Polo, Luis And Berrueta, Diego. Measurement units ontology. Tech. rep.
41. Project REWERSE: Reasoning on the Web WITH RULES AND SEMANTICS. Ontology of transportation networks, deliverable a1-d4. Tech. rep.

42. Renold, A. Pravin And Rani, R. Joshi. An Internet based RFID library management system. In Proceedings of Interntional Conference on information and Communication technologies (2013).

43. Rimal, Bhaskar Prasad AND Choi, Eunmi AND Lumb, IAN. A Taxonomy and Survey of Cloud Computing Systems. In Proceedings of Fifth Interntional Joint Conference on INC, IMS and IDC (2009).

44. R.K., BALAN AND ET AL. mFerio: the design and evaluation of a peer-to-peer mobile payment system. In Proceedings of Interntional Conference on Mobile systems, applications, and services (2009).

45. SANCHEZ, L. AND ET AL. SmartSantander: Experimentation and service provision in the smart city. In Proceedings of Interntional Conference on Wireless Personal Multimedia Communications (2013).

46. Schiele, G., Soldatos, J., And Mitton, N. Moving Towards Interoperable Internet-of-Things Deployments in Smart Cities. ERCIM News 2014, 98 (2014).

47. Sharma, Meenakshi and Siddiqui, Adil. RFID based mobiles: Next generation applications. In Proceedings of Interntional Conference on Information Management and Engineering (2010).

48. TAHERKORDI, AMIRHOSEIN AND ELIASSEN, FRANK. Restful service development for resourceconstrained environments. In REST: From Research to Practice. 2011.

49. The W3C Semantic Sensor Network InCUBATOR GROUP. W3C SSN Incubator Group Review of Sensor and Observation Ontologies. Tech. rep.

50. TheOdoridis, E. AND MYlonas, G. AND Chatzigiannakis, I. Developing an iot smart city framework. In Proceedings of Fourth International Conference on Information, Intelligence, Systems and Applications (July 2013), pp. 1-6.

51. WANG, YANGYANG AND Zhou, Yanhui. Cloud architecture based on Near Field Communication in the smart city. In Proceedings of Interntional Conference on Computer Science \& Education (2012).

52. Xively. https://xively.com.

53. Yuriyama, MadoKa And Kushida, TAKaYUKI. Sensor-Cloud Infrastructure - Physical Sensor Management with Virtualized Sensors on Cloud Computing. In Proceedings of Interntional Conference on Network-Based Information Systems (2010).

54. Zhou, Minqi AND Zhang, Rong AND Zeng, DADAN AND QIAN, WeInING. Services in the Cloud Computing era: A survey. In Proceedings of Universal Communication Symposium (2010). 